\title{
Unified treatment of the Coulomb and harmonic oscillator potentials in $D$ dimensions
}

\author{
G. Lévai, B. Kónya and Z. Papp \\ Institute of Nuclear Research of the Hungarian Academy of Sciences, \\ P. O. Box 51, H-4001 Debrecen, Hungary
}

\begin{abstract}
Quantum mechanical models and practical calculations often rely on some exactly solvable models like the Coulomb and the harmonic oscillator potentials. The $D$ dimensional generalized Coulomb potential contains these potentials as limiting cases, thus it establishes a continuous link between the Coulomb and harmonic oscillator potentials in various dimensions. We present results which are necessary for the utilization of this potential as a model and practical reference problem for quantum mechanical calculations. We define a Hilbert space basis, the generalized Coulomb-Sturmian basis, and calculate the Green's operator on this basis and also present an SU(1,1) algebra associated with it. We formulate the problem for the one-dimensional case too, and point out that the complications arising due to the singularity of the onedimensional Coulomb problem can be avoided with the use of the generalized Coulomb potential.
\end{abstract}

PACS numbers: 03.65.Fd, 03.65.Ge, 03.65.Nk 


\section{INTRODUCTION}

Local potentials have been used to model the interactions of the subatomic world ever since the introduction of quantum mechanics. Some of these (like the Coulomb potential) do not differ essentially from the forces observed in nature, while most of them (like the harmonic oscillator, for example) represent approximations of the actual physical situation. The potential shape, defined by the potential type and the parameters in it is usually chosen in a way that reflects the physical picture our intuition associates with the problem; therefore we can define attractive or repulsive, short-range or long-range potentials, etc.

Some of the potentials used in quantum mechanics are exactly solvable. This means that the energy eigenvalues, the bound-state wave functions and the scattering matrix can be determined in closed analytical form. The range of these potentials has been extended considerably in the recent years by investigations inspired by supersymmetric quantum mechanics (SUSYQM), for example. (See Refs. [1] for recent reviews.) Most of the solvable potentials are one-dimensional problems, which means that they are defined on the $(-\infty, \infty)$ domain (or on some finite interval of it), or they can be reduced to radial problems in higher spatial dimensions, in which case they are confined to the positive real axis. The simplest exactly solvable potentials are those belonging to the so-called shape-invariant class [2]. Many of the most well-known potentials, like the Coulomb, harmonic oscillator, Morse, PöschlTeller, etc. potentials belong to this class. Usually 12 potentials are mentioned to have the property of shape-invariance [3], although two of them are just alternative forms of other shape-invariant potentials.

A rather more general potential type is that of the Natanzon potentials [4], the solutions of which can be reduced to a single hypergeometric or confluent hypergeometric function. These potentials are written as complicated six-parameter expressions of the coordinate, and their energy eigenvalues are given by an implicit formula. Although these problems are solvable exactly in principle, only some specific Natanzon potentials have been studied in detail [5 8], because the formalism is getting more and more complicated with the increasing number of parameters handled. Some of the Natanzon potentials contain certain shapeinvariant potentials as special cases: the Ginocchio potential [6] can be considered as a generalization of the Pöschl-Teller potential, while the generalized Coulomb potential [8] is a simultaneous extension of the Coulomb and the harmonic oscillator potentials. There are also further potentials beyond the Natanzon class. These include, for example quasi-exactly solvable (QES) potentials [9], conditionally exactly solvable (CES) potential [10] and the SUSYQM partners of Natanzon-class potentials [11], of which certain CES potentials form a subclass [12].

The study of exactly solvable potentials represents an interesting field of mathematical physics in itself, but results from this area are also essential for the description of realistic physical problems. More and more interactions can be modeled by making advantage of the rather flexible potential shapes offered by exactly solvable potentials. The solutions of these can be applied directly, or they can be combined with numerical calculations. In the simplest case analytical calculations can aid numerical studies in areas where numerical techniques might not be safely controlled. This is the case, for example, when bound-state wave functions with arbitrary node numbers are required, for certain singular potentials, or for complex potentials [13]. As the next level of complexity, analytical solutions can supply 
a basis for numerical calculations.

Virtually all quantum mechanical methods rely in some respect on analytically solvable potentials. Very often their wave function solutions are used as a Hilbert-space basis. More powerful methods can be constructed if we select a basis which allows the exact analytical calculation of the Green's operator of an analytically solvable potential. Some time ago by utilizing the Coulomb-Sturmian functions as basis functions the Coulomb Green's operator was calculated analytically and a quantum mechanical approximation method was developed [14. Recently this method was extended for approaching the really challenging three-body Coulomb problem [15]. We note here that in this work the exact analytical representation of the Coulomb Green's operator on the Coulomb-Sturmian basis was essential. In our recent work [16] we showed that if on some basis representation the Hamiltonian possesses an infinite tridiagonal matrix (J-matrix) form then its resolvent can be given in terms of continued fractions which can be calculated analytically on the whole complex plane. This theorem was exemplified by the Coulomb and harmonic oscillator Green's operators.

The aim of the present work is to demonstrate that various techniques developed for the simple shape-invariant potentials, like the Coulomb and harmonic oscillator potentials, can be generalized for certain Natanzon-class potentials too without making the formalism significantly more involved, and that these more general potentials can help our understanding of both practical and theoretical problems. The actual example we consider here is the generalized Coulomb potential [8], which is the member of the Natanzon confluent potential class [17. This potential is Coulomb-like asymptotically, while its short-range behavior depends on the parameters: it can be finite or singular as well at the origin. Its shape therefore can approximate various realistic problems, such as nuclear potentials with relatively flat central part, or atomic potentials that incorporate the effect of inner closed shells by a phenomenological repulsive core.

An advantageous feature of the generalized Coulomb problem is that the equivalent of the Coulomb-Sturmian basis can be defined for it, together with all its implications. For example, the method of calculating the matrix elements of the Green's operator as described in Ref. [16] for the Coulomb and the harmonic oscillator potentials can be extended to this case too, so that the above two problems appear in the present treatment as special cases.

Using a particular differential realization, an $\mathrm{SU}(1,1)$ algebra can also be associated with this potential, which reduces to known $\mathrm{SU}(1,1)$ algebras in the Coulomb and harmonic oscillator limits.

Another important feature of this potential is that it can be formulated as a onedimensional problem, but it can avoid the pathologic features of the one-dimensional Coulomb problem, which arise due to the $|x|^{-1}$-like singularity. Since this potential can be chosen to be finite at $x=0$, it offers a convenient basis for calculations which require the use of a one-dimensional Coulomb-like problem.

The arrangement of this paper is the following. In Section $\llbracket$ we give the basic formalism for the generalized Coulomb problem by recapitulating and extending former results. Section III contains the main results of the paper: i) the discussion of the Coulomb-oscillator connection using the two limiting cases of the potential, ii) the determination of the matrix elements of the Green's operator, iii) the description of an $\mathrm{SU}(1,1)$ algebra associated with the problem and $i v$ ) discussion on the one-dimensional case, which elucidates the complications related to the singularity of the one-dimensional Coulomb problem. Finally, we 
summarize the results and discuss further possibilities in Sect. IV].

\section{THE GENERALIZED COULOMB POTENTIAL IN $D$ DIMENSIONS}

Let us consider the Schrödinger equation in $D$ spatial dimensions with a potential that depends only on the radial variable $r$. Separating the angular variables from the wave function we obtain the radial Schrödinger equation

$$
\hat{H}_{0} \psi(r) \equiv\left(-\frac{\mathrm{d}^{2}}{\mathrm{~d} r^{2}}+\frac{1}{r^{2}}\left(l+\frac{D-3}{2}\right)\left(l+\frac{D-1}{2}\right)+v(r)\right) \psi(r)=\epsilon \psi(r),
$$

where $v(r) \equiv 2 m \hbar^{-2} V(r)$ and $\epsilon \equiv 2 m \hbar^{-2} E$. The centrifugal term, which depends on the angular momentum $l$ originates from the kinetic term, i.e. from the $D$-dimensional Laplace operator after separating the angular variables. The bound-state wave functions solving (1) are normalized as

$$
\int_{0}^{\infty}|\psi(r)|^{2} \mathrm{~d} r=1
$$

Here we recapitulate the results obtained for the generalized Coulomb potential in Ref. [8] by adapting it to the $D$-dimensional case. Changing slightly the notation used in Ref. [8] we define the generalized Coulomb potential as

$$
\begin{aligned}
v(r)= & -\frac{1}{r^{2}}\left(l+\frac{D-3}{2}\right)\left(l+\frac{D-1}{2}\right)+\left(\beta-\frac{1}{2}\right)\left(\beta-\frac{3}{2}\right) \frac{C}{4 h(r)(h(r)+\theta)} \\
& -\frac{q}{h(r)+\theta}-\frac{3 C}{16(h(r)+\theta)^{2}}+\frac{5 C \theta}{16(h(r)+\theta)^{3}},
\end{aligned}
$$

where $h(r)$ is defined [8] in terms of its inverse function

$$
r=r(h)=C^{-\frac{1}{2}}\left(\theta \tanh ^{-1}\left(\left(\frac{h}{h+\theta}\right)^{\frac{1}{2}}\right)+(h(h+\theta))^{\frac{1}{2}}\right) .
$$

The $h(r)$ function maps the $[0, \infty)$ half axis onto itself and can be approximated with $h(r) \simeq C^{\frac{1}{2}} r$ and $h(r) \simeq C r^{2} /(4 \theta)$ in the $r \rightarrow \infty$ and $r \rightarrow 0$ limits, respectively.

Bound states are located [8] at

$$
\epsilon_{n}=-\frac{C}{4} \rho_{n}^{2}
$$

where

$$
\rho_{n}=\frac{2}{\theta}\left(\left((n+\beta / 2)^{2}+\frac{q \theta}{C}\right)^{\frac{1}{2}}-(n+\beta / 2)\right)
$$

The bound-state wave functions can be written in terms of associated Laguerre polynomials as 
$\psi_{n}(r)=C^{\frac{1}{4}} \rho_{n}^{\frac{\beta+1}{2}}\left(\frac{\Gamma(n+1)}{\Gamma(n+\beta)\left(2 n+\beta+\rho_{n} \theta\right)}\right)^{1 / 2}(h(r)+\theta)^{\frac{1}{4}}(h(r))^{\frac{2 \beta-1}{4}} \exp \left(-\frac{\rho_{n}}{2} h(r)\right) L_{n}^{(\beta-1)}\left(\rho_{n} h(r)\right)$

Potential (3) clearly carries angular momentum dependence: its first term merely compensates the centrifugal term arising from the kinetic term of the Hamiltonian. Its second term also has $r^{-2}$-like singularity (due to $h^{-1}(r)$ ), and as it will be demonstrated later, it cancels the angular momentum dependent term in the two important limiting cases that recover the $D$-dimensional Coulomb and the harmonic oscillator potentials. The third term of (3) represents an asymptotically Coulomb-like interaction, while the remaining two terms behave like $r^{-2}$ and $r^{-3}$ for large values of $r$.

In the remaining part of this Section we consider the specific case of $D=3$ and $l=0$. The $S$ matrix of the generalized Coulomb potential can be derived in complete analogy with that of the Coulomb problem for $D=3$. Although this can only be done exactly for $l=0$, the singular term imitating the centrifugal term in Eq. (3) can be defined to be part of the potential. Using the notation of Ref. [18] the equivalent of the general regular and irregular solutions are

$$
\varphi^{(G)}(k, r)=C^{-\frac{\beta}{4}}(h(r)+\theta)^{\frac{1}{4}}(h(r))^{\frac{2 \beta-1}{4}} \exp \left(-\frac{\rho}{2} h(r)\right) \Phi\left(\frac{\beta}{2}+\mathrm{i} \nu, \beta ; \rho h(r)\right)
$$

and

$$
f^{(G)}(k, r)=\rho^{-\frac{\beta}{4}} \mathrm{e}^{\frac{\nu \pi}{2}}(h(r)+\theta)^{\frac{1}{4}}(h(r))^{\frac{2 \beta-1}{4}} \exp \left(-\frac{\rho}{2} h(r)\right) \Psi\left(\frac{\beta}{2}+\mathrm{i} \nu, \beta ; \rho h(r)\right)
$$

where $\Phi(a, c ; z)$ and $\Psi(a, c ; z)$ are the regular and the irregular confluent hypergeometric functions [19], and $\rho$ and $\nu$ have to be chosen as

$$
\rho \equiv \rho(k)=-\frac{2 \mathrm{i} k}{C^{1 / 2}}, \quad \mathrm{i} \nu \equiv \mathrm{i} \nu(k)=\frac{\rho \theta}{4}-\frac{q}{C \rho} .
$$

The functions (8) and (9) reduce to $\varphi^{(c)}(k, r)$ and $f^{(c)}(k, r)$ of Ref. [18] in the $\theta \rightarrow 0$ Coulomb limit. The $S$ matrix for $l=0$ is then expressed as

$$
S_{0}(k)=(-1)^{\frac{\beta}{2}+1} \frac{\Gamma\left(\frac{\beta}{2}+\mathrm{i} \nu\right)}{\Gamma\left(\frac{\beta}{2}-\mathrm{i} \nu\right)} .
$$

The extra phase factor appears because of the $r^{-2}$-type singular term, which is now defined to be part of the potential. In the Coulomb limit this expression becomes part of the centrifugal term, which is dealt with separately.

The long-range behavior of potential (3) suggests its use in problems associated with the electrostatic field of some charge distribution. The deviation from the Coulomb potential close to the origin can be viewed as replacing the point-like charge with an extended charged object. The relevant charge density is readily obtained from the potential using

$$
\rho(r)=-\frac{\hbar^{2}}{8 \pi m \mathrm{e}} \Delta v(r)
$$


Choosing $D=3$ and neglecting the terms singular at the origin by making the choice $l=0$ and $\beta=1 / 2$ or $3 / 2$ we get

$$
\begin{aligned}
\rho(r)= & -\frac{\hbar^{2}}{8 \pi m \mathrm{e}}\left[-\frac{2 q C}{(h(r)+\theta)^{3}}+\frac{20 q C \theta-9 C^{2}}{8(h(r)+\theta)^{4}}+\frac{81 C^{2} \theta}{16(h(r)+\theta)^{5}}-\frac{135 C^{2} \theta^{2}}{32(h(r)+\theta)^{6}}\right. \\
& \left.+\frac{2 C^{1 / 2}}{r}\left(\frac{h(r)}{h(r)+\theta}\right)^{1 / 2}\left(\frac{q}{(h(r)+\theta)^{2}}+\frac{3 C}{8(h(r)+\theta)^{3}}-\frac{15 C \theta}{16(h(r)+\theta)^{4}}\right)\right] .
\end{aligned}
$$

In Figs. 1 and 2 we present examples for the actual shape of potential (3) and the corresponding charge distribution (13) for various values of the parameters. It can be seen that this potential is suitable for describing the Coulomb field of extended objects. It is a general feature of potential (3) that for small values of $\theta$ a (finite) positive peak appears near the origin, which also manifests itself in a repulsive "hard core", corresponding to a region with positive charge density (see Fig. 2).

\section{DISCUSSION}

\section{A. The Coulomb and harmonic oscillator limits}

As described in Ref. [8], the introduction of the generalized Coulomb problem was inspired by a simple method of transforming the Schrödinger equation into the differential equation of the confluent hypergeometric function. The bound-state solutions of these problems contain associated Laguerre polynomials. The three most well-known examples of this type are the Coulomb, the harmonic oscillator and the Morse potentials, which are all members of the shape-invariant potential class [2]. These three potentials are recovered by specific choices of the variable transformation reducing the Schrödinger equation to the differential equation of the associated Laguerre polynomials. This is also reflected by the structure of the function $h(r)$ in Eq. ( $/ 1)$ : when $h(r)$ is proportional to $r$ and $r^{2}$, one obtains the Coulomb problem and the harmonic oscillator potential, respectively. These limits can readily be realized by specific choices of the parameters in Eq. (4): the first one follows from the $\theta \rightarrow 0$ limit [8], while the second one is reached by taking $\theta \rightarrow \infty$, while keeping $C / \theta \equiv \tilde{C}$ constant 20.

Besides taking the $\theta \rightarrow 0$ limit, the Coulomb problem in $D$-dimensions is recovered from Eq. (3) by the $\beta=2 l+D-1$ and $C^{-\frac{1}{2}} q=2 m Z \mathrm{e}^{2} / \hbar^{2}$, choices: the third term of (3) becomes the Coulomb term, the fifth one vanishes, while the other three all become proportional with $r^{-2}$ and cancel out completely.

In order to reach the oscillator limit one also has to redefine the potential (3) and the energy eigenvalues by adding $q / \theta$ to both. (This is equivalent to $\tilde{C} \tilde{D} \theta$ in the notation of Ref. [20].) This choice simply represents resetting the energy scale: $\epsilon=0$ corresponds to $v(r \rightarrow \infty)$ for the Coulomb problem, and to $v(r=0)$ for the harmonic oscillator. (Note that the energy eigenvalues also have different signs in the two cases.) Besides $C / \theta=\tilde{C}$, the $\tilde{q} \equiv q / \theta^{2}$ parameter also has to remain constant in the $\theta \rightarrow \infty$ transition here. The potential thus adapted to the harmonic oscillator limit reads 


$$
\begin{aligned}
\tilde{v}(r) \equiv v(r)+q \theta= & -\frac{1}{r^{2}}\left(l+\frac{D-3}{2}\right)\left(l+\frac{D-1}{2}\right)+\left(\beta-\frac{1}{2}\right)\left(\beta-\frac{3}{2}\right) \frac{\tilde{C}}{4 h(r)\left(1+\frac{h(r)}{\theta}\right)} \\
& -\frac{\tilde{q} h(r)}{1+\frac{h(r)}{\theta}}-\frac{3 \tilde{C}}{16 \theta} \frac{1}{\left(1+\frac{h(r)}{\theta}\right)^{2}}+\frac{5 \tilde{C}}{16 \theta} \frac{1}{\left(1+\frac{h(r)}{\theta}\right)^{3}} .
\end{aligned}
$$

The harmonic oscillator potential is recovered from (14) by the $\beta=l+D / 2$ and $\tilde{C} \tilde{q}=$ $(2 m \omega / \hbar)^{2}$ choice. The two last terms in (14) vanish, the first and the second cancel out, while the third one reproduces the harmonic oscillator potential. The new form of the energy eigenvalues is

$$
\tilde{\epsilon}_{n} \equiv \epsilon_{n}+q / \theta=\tilde{C}(2 n+\beta)\left(\left(\frac{1}{\theta^{2}}\left(n+\frac{\beta}{2}\right)^{2}+\frac{\tilde{q}}{\tilde{C}}\right)^{\frac{1}{2}}-\frac{1}{\theta}\left(n+\frac{\beta}{2}\right)\right)
$$

which indeed, reduces to the $\tilde{\epsilon}_{n}=(2 m \omega / \hbar)(2 n+l+D / 2)$ oscillator spectrum in the $\theta \rightarrow \infty$ limit. The wave functions (17) are unchanged, except for the redefinition of the parameters.

Note that the generalized Coulomb problem establishes a link between the Coulomb problem and the harmonic oscillator potential in different spatial dimensions. This is best seen by inspecting the wave functions (7). If the $\beta-1$ parameter of the associated Laguerre polynomial is required to be the same in the two limits, we get an interrelation between the value of the angular momentum and the spatial dimension to be used for the Coulomb and the harmonic oscillator case:

$$
l^{O}+\frac{D^{O}}{2}=2 l^{C}+D^{C}-1 .
$$

Considering $l^{O}=2 l^{C}$, Eq. (16) implies $D^{O}=2 D^{C}-2$ which establishes link between the $\left(D^{C}, D^{O}\right)=(2,2),(3,4),(4,6),(5,8), \ldots$ etc. pairs. (Of these the $(3,4)$ pair corresponds to the Kustaanheimo-Stiefel transformation [21].) This case is called the "direct map" between the Coulombic and oscillator solutions in Ref. [22], while with the $l^{O}=2 l^{C}+\lambda$ choice the "general map" can be recovered. These results suggest that the generalized Coulomb potential can be used to formulate a continuous transition between the Coulomb and harmonic oscillator potentials, as opposed to the usual procedure that employs a unique variable and parameter transformation to reach this goal.

\section{B. The generalized Coulomb-Sturmian basis and the matrix elements of the Green's operator}

The generalized Coulomb-Sturmian equation which depends on $n$ as a parameter, has similar structure to the eigenvalue equation in Eq. (四) with potential (3)

$$
\begin{aligned}
\hat{X} \phi(\rho, r) \equiv & {\left[-\frac{\mathrm{d}^{2}}{\mathrm{~d} r^{2}}-\frac{3 C}{16(h(r)+\theta)^{2}}+\frac{5 C \theta}{16(h(r)+\theta)^{3}}+\frac{C\left(\beta-\frac{1}{2}\right)\left(\beta-\frac{3}{2}\right)}{4 h(r)(h(r)+\theta)}\right.} \\
& \left.-\left(\frac{\rho^{2} \theta}{4}+\rho\left(n+\frac{\beta}{2}\right)\right) \frac{C}{h(r)+\theta}+\frac{C}{4} \rho^{2}\right] \phi(\rho, r)=0,
\end{aligned}
$$


and is solved by the generalized Coulomb-Sturmian (GCS) functions

$$
\langle r \mid n\rangle \equiv \phi_{n}(\rho, r)=\left(\frac{\Gamma(n+1)}{\Gamma(n+\beta)}\right)^{1 / 2}(\rho h(r)+\rho \theta)^{\frac{1}{4}}(\rho h(r))^{\frac{2 \beta-1}{4}} \exp \left(-\frac{\rho}{2} h(r)\right) L_{n}^{(\beta-1)}(\rho h(r)) .
$$

Here $\rho$ is a parameter characterizing the generalized Coulomb-Sturmian basis. The GCS functions, being solutions of a Sturm-Liouville problem, have the property of being orthonormal with respect to the weight function $C^{\frac{1}{2}}(h(r)+\theta)^{-1}$. Introducing the notation $\langle r \mid \widetilde{n}\rangle \equiv \phi_{n}(\rho, r) C^{\frac{1}{2}}(h(r)+\theta)^{-1}$ the orthogonality and completeness relation of the GCS functions can be expressed as

$$
\langle n \mid \widetilde{n}\rangle=\delta_{n n^{\prime}}
$$

and

$$
1=\sum_{n=0}^{\infty}|\widetilde{n}\rangle\left\langle n\left|=\sum_{n=0}^{\infty}\right| n\right\rangle\langle\widetilde{n}|
$$

Straightforward calculation shows that both the overlap of two GCS functions and the $\left\langle n^{\prime}\left|\hat{H}_{0}\right| n\right\rangle$ matrix element can be expressed as a tridiagonal matrix, therefore the matrix elements of the $\epsilon-\hat{H}_{0}$ operator also have this feature:

$$
\begin{aligned}
\left\langle n\left|\epsilon-\hat{H}_{0}\right| n^{\prime}\right\rangle & =\delta_{n n^{\prime}}\left(\frac{\epsilon}{C^{\frac{1}{2}} \rho}(2 n+\beta-\rho \theta)-\frac{C^{\frac{1}{2}} \rho}{4}\left(-\frac{4 q}{C \rho}+(2 n+\beta)\right)\right) \\
& -\delta_{n n^{\prime}+1}(n(n+\beta-1))^{\frac{1}{2}}\left(\frac{\epsilon}{C^{\frac{1}{2}} \rho}+\frac{C^{\frac{1}{2}} \rho}{4}\right)-\delta_{n n^{\prime}-1}((n+1)(n+\beta))^{\frac{1}{2}}\left(\frac{\epsilon}{C^{\frac{1}{2}} \rho}+\frac{C^{\frac{1}{2}} \rho}{4}\right) .
\end{aligned}
$$

This means that similarly to the $D$-dimensional Coulomb and harmonic oscillator potential the matrix elements of the Green's operator can be determined by using continued fractions, as described in Ref. [16]. The present results, therefore, extend the applicability of this method to a new potential problem. The formulae presented here reduce to those in [16] in the appropriate limits discussed in Subsection limits. The role of the Coulomb-Sturm parameter $b$ used in [16] is now played by $C^{\frac{1}{2}} \rho / 2$.

\section{Algebraic aspects}

Here we show that an $\mathrm{SU}(1,1)$ algebra can be associated with the generalized Coulomb problem. The generators of this algebra are

$$
\begin{aligned}
& \hat{J}_{3}=\frac{h+\theta}{C \rho} \hat{X}+\left(n+\frac{\beta}{2}\right), \quad \hat{J}_{1}=\hat{J}_{3}-\frac{\rho}{2} h, \\
& \hat{J}_{2}=-\frac{\mathrm{i}}{C^{1 / 2}}(h(h+\theta))^{1 / 2} \frac{\mathrm{d}}{\mathrm{d} r}-\frac{\mathrm{i} \theta}{4(h+\theta)},
\end{aligned}
$$


an they satisfy the commutation relations

$$
\left[\hat{J}_{1}, \hat{J}_{2}\right]=-\mathrm{i} \hat{J}_{3} \quad\left[\hat{J}_{2}, \hat{J}_{3}\right]=\mathrm{i} \hat{J}_{1} \quad\left[\hat{J}_{3}, \hat{J}_{1}\right]=\mathrm{i} \hat{J}_{2}
$$

As can be seen from Eq. (17), $\hat{J}_{3}$ is diagonal in the basis (18) with eigenvalues $m=n+\frac{\beta}{2}$. The elements of this basis can then be associated with the infinite-dimensional discrete unitary irreducible representation of $\mathrm{SU}(1,1)$ called [23] discrete principal series $D_{j}^{+}$, for which the allowed values of $m$ are

$$
m=-j,-j+1,-j+2, \ldots
$$

with $j$ being negative. It is natural then to identify $j$ as $j=-\frac{\beta}{2}$. Direct calculations show that the ladder operators connect the neighboring members of this basis:

$$
\begin{gathered}
\hat{J}_{+} \phi_{n}(\rho, r) \equiv\left(\hat{J}_{1}+\mathrm{i} \hat{J}_{2}\right) \phi_{n}(\rho, r)=[(n+1)(n+\beta)]^{1 / 2} \phi_{n+1}(\rho, r), \\
\hat{J}_{-} \phi_{n}(\rho, r) \equiv\left(\hat{J}_{1}-\mathrm{i} \hat{J}_{2}\right) \phi_{n}(\rho, r)=[n(n+\beta-1)]^{1 / 2} \phi_{n-1}(\rho, r) .
\end{gathered}
$$

We find that the eigenvalues of the Casimir invariant

$$
\hat{C}_{2}=\hat{J}_{3}^{2}-\hat{J}_{1}^{2}-\hat{J}_{2}^{2}
$$

are $\frac{\beta}{2}\left(\frac{\beta}{2}-1\right)=j(j+1)$, as expected, and that they set the strength of the fourth term in (17). For $\theta \neq 0$ this is the only singular term and it behaves like $\gamma r^{-2}$ with $\gamma=\left(\beta-\frac{1}{2}\right)\left(\beta-\frac{3}{2}\right)=$ $4 j(j+1)+\frac{3}{4}$. It is interesting to inspect the allowed values of $\gamma$ for the different unitary irreducible representations of $\mathrm{SU}(1,1)$. For the discrete principal series $D_{j}^{+} \beta>1$ holds, which always secures $-\frac{1}{4}<\gamma$, i.e. the potential has repulsive or "weakly attractive" 24] $r^{-2}$-type singularity. For the supplementary series [23] $-\frac{1}{2}<j<0$ holds, which results in $-\frac{1}{4}<\gamma<\frac{3}{4}$. This is exactly the domain where both independent solutions are square integrable at the origin [24]: for $0<\gamma<\frac{3}{4}$ one of these vanishes at $r=0$ and the other one is infinite there, while for $-\frac{1}{4}<\gamma<0$ for both solutions vanish at $r=0$. ¿From (18) it is seen that solutions regular and irregular at the origin correspond to $\beta>\frac{1}{2}$ and $\beta<\frac{1}{2}$. This seems to indicate that one regular solution (with $\beta>1$ ) is associated with $D_{j}^{+}$, while the second square integrable solution, which is either regular or infinite at the origin (depending on $\beta$ ) might be related to the supplementary series, for which $0<\beta<1$ holds. For the sake of completeness we note that for the continuous series 23 a $C_{k}^{0}$ and $C_{k}^{1 / 2}$, $j=-\frac{1}{2}+\mathrm{i} k(k>0$, real $)$ is valid, which results in the strongly singular $\gamma<-\frac{1}{4}$ case. The solutions then oscillate infinitely near the origin and are unbounded from below [24], which can be interpreted as the falling of the particle into the center of attraction [25]. The situation is similar to that described in Ref. [26] for the $v(x)=\gamma \sin ^{-2} x$ potential: the various unitary irreducible representations of the $\mathrm{SU}(1,1)$ spectrum generating group there also corresponded to different types of singularities.

We note that we analyzed the singularities of the generalized Coulomb-Sturmian equation (17) and its solutions (18), but similar considerations of the physical potential (3) and its solutions can also be performed taking $D=3$ and $l=0$. The algebraic construction, however, does not apply to this latter problem. This is because the bound-state solutions 
(7) pick up extra $n$-dependence through $\rho_{n}$, which is not accessible for the ladder operators otherwise changing $n$ as in Eqs. (25) and (26).

The present realization of the $\mathrm{SU}(1,1)$ algebra is a special case of that described in Ref. [17] in relation with the Natanzon confluent potentials. Considering the Coulomb and harmonic oscillator limits discussed in Subsection IIIA and setting the dimension to $D=3$, the generators reduce to the forms presented for the two problems separately in Ref. [27. We note that the spectrum generating algebra associated this way with the radial harmonic oscillator problem in three dimensions is different from the two-parameter realization of the $\mathrm{SU}(1,1)$ algebra discussed in Refs. [28,29], because the ladder operators there are linear differential operators and the Hamiltonian is related to the Casimir invariant, while here the ladder operators are second-order differential operators and the Hamiltonian is essentially a linear function of generator $\hat{J}_{3}$.

Finally, we note that similar limiting cases of other Natanzon-class potentials can also be treated in terms of $\mathrm{SU}(1,1)$ algebras. In Ref. [29] an $\mathrm{SU}(1,1)$ algebra associated with the Ginocchio potential [5] was analyzed, and it was shown that in two different limiting cases, which both result in the Pöschl-Teller potential, two different algebras can be recovered from the original one: the first being a spectrum generating algebra and the second a potential algebra.

\section{The one-dimensional case}

The formalism developed previously is valid for $D=1$ too, nevertheless, some particular properties of one-dimensional problems justify a separate treatment of this case. First, the implicit definition of the $h(r)$ function in Eq. (4) has to be extended to negative values of $r$, which we now denote with $x$. Using the notation of (田), we can write that $x=r(h)$ for $x \geq 0$ and $x=-r(h)$ for $x<0$. The normalization of the wave functions in (7) also has to be modified with a factor of $2^{-1 / 2}$, accounting for the fact that the integration now runs from $-\infty$ to $\infty$, but since the potential is symmetric, the bound-state wave functions are either even or odd, therefore $|\psi(x)|^{2}$ is an even function of $x$.

For one-dimensional problems it is natural to set $l$ to 0 besides $D=1$, which eliminates the centrifugal term in (3). Furthermore, in order to avoid $r^{-2}$-like singularities at $x=0$ the second term in (3) also has to be canceled by setting $\beta$ to either $1 / 2$ or to $3 / 2$. Elementary calculations show that the latter choice corresponds to bound-state wave functions that vanish at $x=0$, and essentially represent physical solutions of the problem in higher dimensions as well, while the former choice recovers solutions that do not vanish in general at $x=0$. These two possibilities can naturally be interpreted as odd and even solutions of the one-dimensional potential problem. Furthermore, for $x \geq 0$ the two types of wave functions can be rewritten into a common notation (up to a sign) by making use of the relation of associated Laguerre and Hermite polynomials, when the former ones have $\alpha=1 / 2$ or $\alpha=-1 / 2$ as parameters [30]: 
$\psi_{N}^{(D=1)}(x)=\frac{C^{\frac{1}{4}} \tilde{\rho}_{N}^{\frac{3}{4}}}{2^{N}\left(\Gamma\left(\frac{N+1}{2}\right) \Gamma\left(\frac{N}{2}+1\right)\left(N+\frac{1}{2}+\theta \tilde{\rho}_{N}\right)^{\frac{1}{2}}\right.}(h(x)+\theta)^{\frac{1}{4}} \exp \left(-\frac{\tilde{\rho}_{N}}{2} h(x)\right) H_{N}\left(\left(\tilde{\rho}_{N} h(x)\right)^{\frac{1}{2}}\right)$.

For $x \leq 0$ the bound-state wave functions satisfy

$$
\psi_{N}^{(D=1)}(-x)= \begin{cases}\psi_{N}^{(D=1)}(x) & \text { for } N=2 n\left(\beta=\frac{1}{2}\right) \\ -\psi_{N}^{(D=1)}(x) & \text { for } N=2 n+1\left(\beta=\frac{3}{2}\right) .\end{cases}
$$

In (28) we defined $\tilde{\rho}_{N}$ as

$$
\tilde{\rho}_{N} \equiv \frac{1}{\theta}\left(\left(\left(N+\frac{1}{2}\right)^{2}+4 \frac{q \theta}{C}\right)^{\frac{1}{2}}-\left(N+\frac{1}{2}\right)\right)
$$

which reduces to $\rho_{\left[\frac{N}{2}\right]}$, where even and odd values of $N$ have to be chosen with $\beta=1 / 2$ and $\beta=3 / 2$, respectively, and the integer part of $N / 2$ corresponds to $n$ used in $\rho_{n}$ in Eq. (6).

An interesting aspect of this potential is that it remains finite at $x=0(v(0)=-q / \theta+$ $\left.C /\left(8 \theta^{2}\right)\right)$ for any finite value of $\theta$, however, a narrow, finite peak appears in the $\theta \rightarrow 0$ limit, which then becomes an attractive $-3 /\left(16 r^{2}\right)$-like singularity in the Coulomb limit. This is due to the fourth term in (3) and it corresponds to a "weak" singularity in the sense that the center of attraction is not strong enough for the particle to become infinitely bound [25]. This finite barrier arising for small, but finite $\theta$ values also introduces the possibility of studying tunneling effects in symmetric potential wells. We also note that besides being finite at $x=0$, potential (3) has continuous derivative there, as can directly be verified.

Based on these features the $D=1$ version of potential (3) can be used to analyze the peculiarities of the one-dimensional Coulomb potential defined as $v^{(c)}=-\mathrm{e}^{2} /|x|$. This problem has been the subject of intensive studies in the past couple of decades, but there is still some controversy in the interpretation of the results (see e.g. [31] for a recent review). The unusual features attributed to this singular problem include degenerate eigenvalues 32 interpreted in terms of a hidden $\mathrm{O}(2)$ symmetry [33], an infinitely bound ground state [32] and continuous bound-state spectrum [34. The last two of these were later found to be based on unacceptable solutions of the Schrödinger equation [35,36], while the unexpected degeneracy was explained by an impenetrable barrier at $x=0$, which separates the problem into two disjoint, non-communicating systems with identical energy spectra [36] and makes even the concept of parity obsolete here [37]. Most authors discussing the one-dimensional Coulomb problem agree that the usual techniques of quantum mechanics alone in dealing with potentials are not sufficient in this case. In Refs. [38] for example self-adjoint extension of the relevant differential operator has been discussed.

The one-dimensional version of potential (3) can be chosen such that it becomes close to non-singular potentials used in the approximation of the true one-dimensional Coulomb potential. In fact, with appropriate choice of $q$ and $\theta$ any desired Coulomb asymptotics and $v(x=0)$ value can be generated. Fig. 1 shows potentials with rounded-off shape near $x=0$ $(\theta=1, q=0.5)$ and also ones close to the Coulomb potential with a cutoff $-\mathrm{e}^{2} /(|x|+a)$ $(\theta=1, q=2.5)$. In contrast with these modified Coulomb potentials, all calculations 
can be performed exactly with (8). In the $\theta \rightarrow 0$ limit the generalized Coulomb potential recovers the one-dimensional Coulomb potential supplemented with the $-\frac{3}{16} x^{-2}$ term. This means that the one-dimensional Coulomb potential cannot be reached exactly, nevertheless, reasonable approximations of it can be given, as it will be demonstrated below.

The odd solutions, of course, vanish at $x=0$, while the even solutions have non-zero value there as long as $\theta>0$ holds. In the $\theta \rightarrow 0$ limit $\psi_{N=2 n}^{(D=1)}(0)$ varies with $\theta^{1 / 4}$, so the even solutions also tend to zero at $x=0$. This is in accordance with the discussion in Subsection [IIC on weakly attractive $\gamma r^{-2}$ type singular potentials on the half line: for $-\frac{1}{4}<\gamma<0$ both independent solutions vanish at the origin, so the wave functions are necessarily zero at $r=0$. If we try to extend the $N=2 n$ solutions (28) in the $\theta=0$ Coulomb limit to the $(-\infty, 0)$ domain we find that due to its $x^{1 / 4}$ type behavior at the origin the derivative of an even wave function would not be continuous anymore.

It is also interesting to compare the Coulomb limit of the one-dimensional generalized Coulomb potential with the true one-dimensional Coulomb potential. Switching off gradually the $\gamma r^{-2}$ term we find that the two types of solutions contain confluent hypergeometric functions of the type $\Phi(-n, c ; z)$ with $c=1+(1+4 \gamma)^{1 / 2}$ and $c=1-(1+4 \gamma)^{1 / 2}$. Starting with $\gamma=-\frac{3}{16}$ we recover the $c=\frac{3}{2}$ and $\frac{1}{2}$ corresponding to the two $c=\beta$ values assigned to the allowed solutions of the one-dimensional generalized Coulomb potential and its Coulomb limit. Taking the $\gamma \rightarrow 0$ limit results in $c=2$ and $c=0$. The first one of these corresponds to the allowed solutions of the one-dimensional Coulomb potential (see e.g. Ref. [39]), while the latter one has to be rejected, because the confluent hypergeometric function $\Phi(a, c ; z)$ is not defined for $c=0$. This shows that even solutions which are allowed for the generalized Coulomb potential in one dimension do not have equivalents in the true one-dimensional Coulomb case.

We also note that the true one-dimensional Coulomb potential can be reached in the $\theta \rightarrow 0$ limit setting $\beta$ to 2 from the beginning. In this case, however, a repulsive $3 /\left(16 r^{2}\right)$ type singularity appears, which cancels out only for $\theta=0$. This repulsive singularity then separates the problem into two parts (with $x>0$ and $x<0$ ) from the beginning, so the potential is not a real one-dimensional one. The $\beta=0$ choice, in principle, also gives the same potential, however, the solutions associated with $\beta=0$ are not defined, as we have shown previously. Using $\beta=\varepsilon$ or $2-\varepsilon$ with $\varepsilon$ being small we can get as close to the true one-dimensional Coulomb potential as we wish, but the repulsive singularity is then present for all $\theta$ values.

The reflection and transmission coefficients can be analyzed using the asymptotic behavior of the general solutions of the one-dimensional problem. These can be chosen to be even and odd functions of $x$. Making use of Eq. (8) the even and the odd solutions can be defined for $x \geq 0$ in terms of $\varphi^{(G)}(k, x)$ for $x \geq 0$, setting $\beta=\frac{1}{2}$ and $\frac{3}{2}$, respectively; and their extension to $x \leq 0$ can be given using a formula similar to Eq. (29). The two solutions are interrelated by Eq. 6.3(3) of Ref. [19]. Due to the symmetric nature of the one-dimensional potential $(v(x)=v(-x))$ it is enough to analyze the asymptotic behavior of the solutions for $x \rightarrow \infty$ : the $x \rightarrow-\infty$ case follows naturally. Straightforward calculations show that the reflection coefficient is

$$
R(k)=\frac{\mathrm{e}^{-\mathrm{i} \pi / 4}}{2}\left(\frac{\Gamma\left(\frac{1}{4}+\mathrm{i} \nu\right)}{\Gamma\left(\frac{1}{4}-\mathrm{i} \nu\right)}-\mathrm{i} \frac{\Gamma\left(\frac{3}{4}+\mathrm{i} \nu\right)}{\Gamma\left(\frac{3}{4}-\mathrm{i} \nu\right)}\right) .
$$


Strong reflection is found for potentials having a (finite) barrier in $x=0$ (like those in Fig. 1 with $\theta=0.01$ ), while more regular shapes (like that in Fig. 1 with $\theta=1$ and $q=2.5$, for example) give weak reflection. The $k$ corresponding to maximal reflection increases as $\theta$ get closer to 0 (i.e. as the barrier at $x=0$ gets higher). $|R(k)|$ is small for very large and very small values of $k$. Fig. 3 presents illustration for $|R(k)|^{2}$ for various values of $\theta$. Our findings on the reflection coefficient of the one-dimensional generalized Coulomb potential seem to support the existence of the space splitting effect [37] valid for the Coulomb potential on one dimension: in situations close to the true Coulomb case (e.g. $\theta \rightarrow 0)|R(k)|^{2}$ is close to 1 , which corresponds to a nearly impenetrable barrier at $x=0$. This is also confirmed by our analysis on other approximations of the one-dimensional Coulomb potential, i.e. those with $\beta=\varepsilon$ or $2-\varepsilon$ above.

Besides its mathematical aspects the one-dimensional Coulomb potential has physical relevance too, in the description of the hydrogen atom in strong magnetic field [40], for example. In such practical calculations it is reasonable to use a non-singular Coulomb-like potential instead of the true one-dimensional Coulomb potential: the finite size of the nucleus can be a justification for this. This means that the one-dimensional Coulomb potential might not be sufficient in such calculations: the basis defined with it simply does not contain evenparity states. In practical calculations therefore the use of bases like that assigned to the generalized Coulomb potential is necessary.

\section{SUMMARY AND OUTLOOK}

We analyzed the generalized Coulomb potential and extended results for the $D$ dimensional Coulomb and harmonic oscillator potentials to this potential too, which includes these two simple problems as special cases. The results presented here have both theoretical and practical significance. The theoretical aspects concern the treatment of Coulomb-like problems in one dimension. Due to the singular nature of the one-dimensional Coulomb potential the usual methods of defining a physically relevant basis are not sufficient in this case. The generalized Coulomb potential avoids these complications and it supplies a basis (including both even and odd functions) which can be used instead of the Coulomb-Sturmian basis, which is otherwise a rather helpful tool in higher dimensions, e.g. the three-body Coulomb problem has been approached this way [15]. We also gave a unification of the $\mathrm{SU}(1,1)$ algebras associated with the Coulomb and harmonic oscillator problems separately.

The practical aspects of our work include the introduction of the generalized CoulombSturmian basis, which we used as a basis for analytic representation of the Green's operator. The generalized Coulomb potential (being the member of the rather general Natanzon potential class) offers potential shapes that can approximate various physical problems more accurately than the conventional textbook potentials. In particular, its long-range Coulomblike behavior makes it a good candidate for describing the Coulomb field of extended (nonpoint-like) charge distributions. It can be applied either directly, or as a basis for numerical calculations. Its direct applications can be envisaged in describing muonic atoms, where it can model the Coulomb field of the nucleus. It can also be combined with short-range potentials in the potential separable expansion formalism [14,[15], for example. Then the Coulombic asymptotics of the interaction between the strongly interacting charged objects 
could be taken care of by the Green's operator and only the short-range (nuclear) interaction would have to be approximated.

Similar extensions of other relatively simple (shape-invariant) potentials also seem possible. The example of the generalized Coulomb potential shows that the techniques developed for these well-known potentials can be extended to more general potentials without much technical difficulty. The generalized potentials "inherit" many important features of the simple potentials, of which they are extensions, and, at the same time, they can be adapted to more complex physical situations, due to their more flexible shape. There are other examples confirming the expectations that seemingly complicated formulae can be evaluated analytically even for highly non-trivial potentials. In Ref. [41], for example, potentials

phase-equivalent with the Ginocchio potential [6] have been determined analytically, using the transformations of supersymmetric quantum mechanics.

Based on the present results we believe that the Natanzon-class potentials, which have been analyzed almost exclusively in theoretical studies until now, can be used in practical applications too.

\section{ACKNOWLEDGEMENTS}

This work has been supported by the OTKA contracts No. F20689 and T17298, and grant No. JF 345/93 of the U.S-Hungarian Science and Technology Joint Fund. 


\section{REFERENCES}

[1] G. Lévai, in Quantum Inversion Theory and Applications, ed. H. V. von Geramb, Lecture Notes in Physics 427 (Springer, Berlin, 1994) p. 107;

F. Cooper, A. Khare and U. Sukhatme, Phys. Rep. 251, 268 (1995);

G. Junker, Supersymmetric Methods in Quantum and Statistical Physics, Berlin, Springer, 1996.

[2] L. E. Gendenshtein, Zh. Eksp. Teor. Fiz. Pis. Red. 38, 299 (1983); (Engl. transl. JETP Lett. 38, 35 (1983)).

[3] G. Lévai, J. Phys. A 22, 689 (1989).

[4] G. A. Natanzon, Teor. Mat. Fiz. 38, 146 (1979).

[5] J. N. Ginocchio, Ann. Phys. (N.Y.) 152, 203 (1984).

[6] J. N. Ginocchio, Ann. Phys. (N.Y.) 159, 467 (1985).

[7] S. Brajamani and C. A. Singh, J. Phys. A 23, 3421 (1990);

G. Lévai, J. Phys. A 24, 131 (1991);

B. W. Williams, J. Phys. A 24, L667 (1991);

B. W. Williams and D. Poulios, Eur. J. Phys. 14, 222 (1993);

B. W. Williams, J. L. Rutherford and G. Lévai, Phys. Lett. A 199, 7 (1995).

[8] G. Lévai and B. W. Williams, J. Phys. A 26, 3301 (1993).

[9] A. K. Ushveridze, Quasi-exactly solvable Models in Quantum Mechanics, Bristol, Institute of Physics Publishing, 1994.

[10] A. de Souza Dutra, Phys. Rev. A 47, R2435 (1993);

R. Dutt, A. Khare and Y. P. Varshni, J. Phys. A 28, L107 (1995).

[11] F. Cooper, J. N. Ginocchio and A. Khare, Phys. Rev. D 36, 2458 (1987).

[12] G. Junker and P. Roy, Phys. Lett. A 232, 155 (1997).

[13] D. Baye, G. Lévai and J.-M. Sparenberg, Nucl. Phys. A 599, 435 (1996).

[14] Z. Papp, J.Phys. A 20, 153 (1987);

Phys. Rev. C 38, 2457 (1988);

Phys. Rev. A 46, 4437 (1992);

Comput. Phys. Commun. 70, 426, 435, 4437 (1992).

[15] Z. Papp and W. Plessas, Phys. Rev. C 54, 50 (1996);

Z. Papp, Phys. Rev. C 55, 1080 (1997).

[16] B. Kónya, G. Lévai and Z. Papp, J. Math. Phys., 38, 4832 (1997).

[17] P. Cordero and S. Salamó, J. Phys. A 24, 5299 (1991).

[18] R. G. Newton, Scattering Theory of Waves and Particles, New York, Heidelberg, Berlin, Springer-Verlag, 1982.

[19] A. Erdélyi (Editor), Higher Transcendental Functions Vol. I, New York, McGraw-Hill, 1953.

[20] G. Lévai, in Proc. 2nd Workshop on Harmonic Oscillators, March 23-25, 1994, Cocoyoc, Mexico; NASA Conf. Publications 3286 (1994), p. 113.

[21] P. Kustaanheimo and E. Stiefel, J. Reine angew. Math. 218, 204 (1965).

[22] V. A. Kostelecký, M. M. Nieto and D. R. Truax, Phys. Rev. D 32, 2627 (1985).

[23] B. G. Wybourne, Classical Groups for Physicists, New York, Wiley, 1974.

[24] W. M. Frank, D. J. Land and R. M. Spector, Rev. Mod. Phys. 43, 36 (1971).

[25] L. D. Landau and E. M. Lifshitz, Quantum Mechanics, Oxford, Pergamon, 1977. 
[26] G. Lévai, in Proceedings of the IVth Wigner Symposium, (Eds. N. M. Atakishiyev, T. H. Seligman and K. B. Wolf), Singapore, World Scientific, 1996; p. 88.

[27] I. L. Cooper, J. Phys. A 26, 1601 (1993).

[28] G. Lévai, J. Phys. A 27, 3809 (1994).

[29] G. Lévai, in Proceedings of the XXIth International Colloquium on Group Theoretical Methods in Physics, Vol. 1, (Eds. H.-D. Doebner, P. Nattermann and W. Scherer), Singapore, World Scientific, 1997; p. 461.

[30] M. Abramowitz and I. Stegun, Handbook of Mathematical Functions, New York, Dover, 1970.

[31] A. N. Gordeyev and S. C. Chhajlany, J. Phys. A 30, 6893 (1997).

[32] R. Loudon, Am. J. Phys. 27, 649 (1959).

[33] L. S. Davtyan, G. S. Pogosyan, A. N. Sissakian and V. M. Ter-Antonyan, J. Phys. A 20, 2765 (1987).

[34] L. K. Haines and D. H. Roberts, Am. J. Phys. 37, 1145 (1969).

[35] M. Andrews, Am. J. Phys. 34, 1194 (1966).

[36] M. Andrews, Am. J. Phys. 44, 1064 (1976).

[37] U. Oseguera and M. de Llano, J. Math. Phys. 34, 4575 (1993).

[38] F. Gesztesy, J. Phys. A: Math. Gen. 13, 867 (1980);

W. Fischer, H. Leschke and P. Müller, J. Math. Phys. 36, 2313 (1995).

[39] R. G. Newton, J. Phys. A 27, 4717 (1994).

[40] H. Ruder, G. Wunner, H. Herold and F. Geyer, Atoms in Strong Magnetic Fields, Berlin, Springer, 1994.

[41] G. Lévai, D. Baye and J.-M. Sparenberg, J. Phys. A 30, 8257 (1997). 


\section{FIGURES}

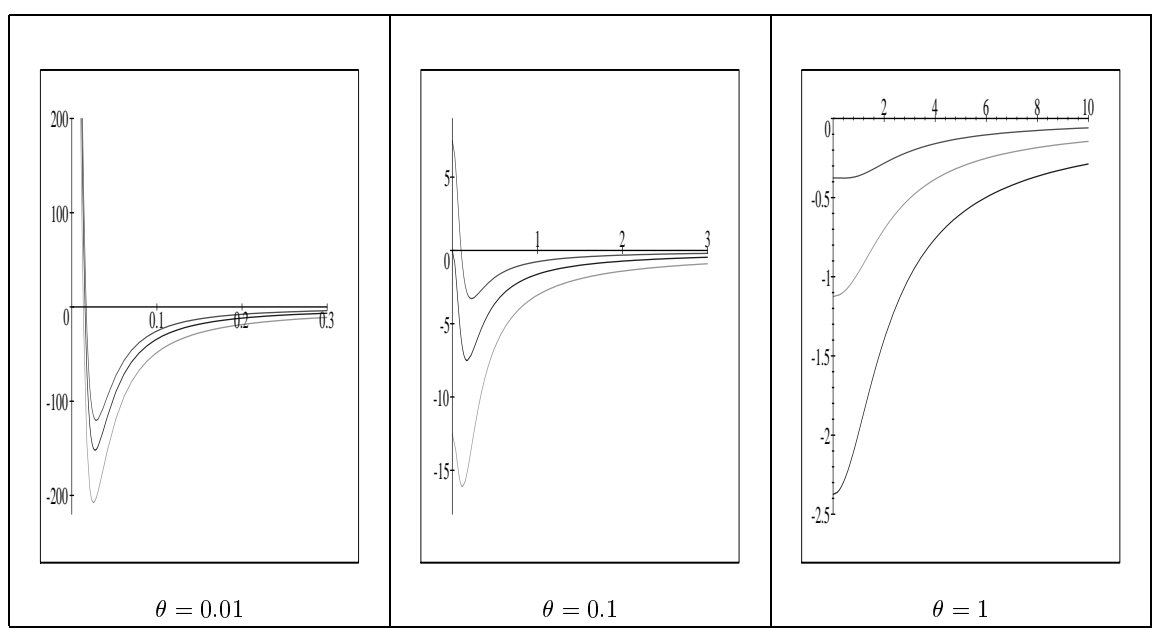

Figure 1: The generalized Coulomb potential for $q=0.5,1.25,2.5 ; \theta=0.01,0.1,1 ; C=1$ and $\beta=3 / 2 . l=0$ and $D=3$ is also implied. In each panel the largest $q$ corresponds to the lowest curve. Note the different scales of the horizontal $(r)$ and the vertical $(v(r))$ axes.

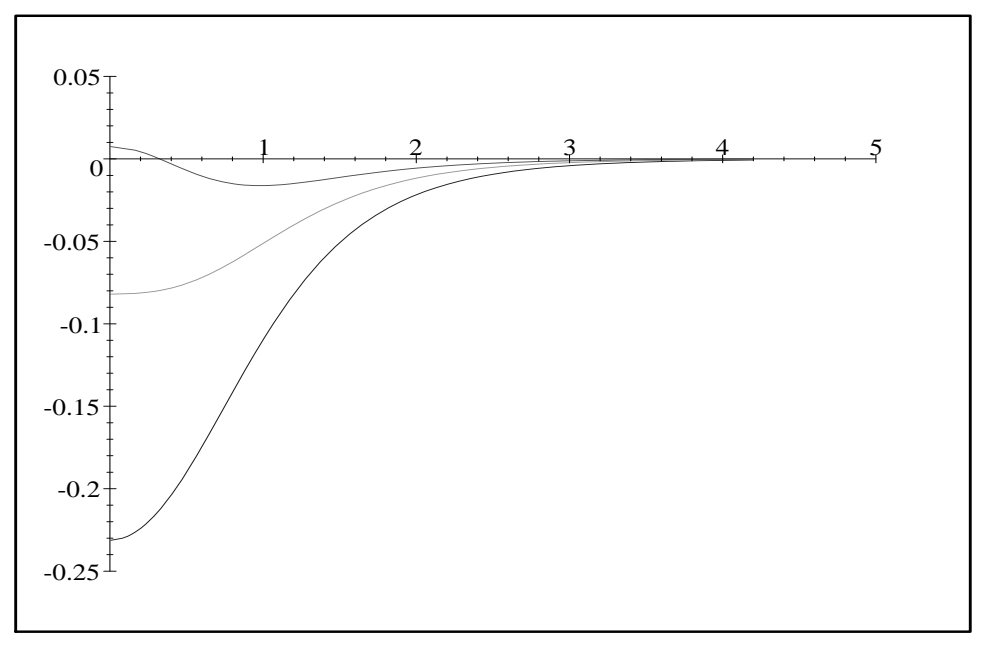

Figure 2: The charge distributions corresponding to the potentials displayed in Fig. 1 for $\theta=1$ and $q=0.5,1.25$, 2.5. (The largest $q$ corresponds to the lowest curve.) 


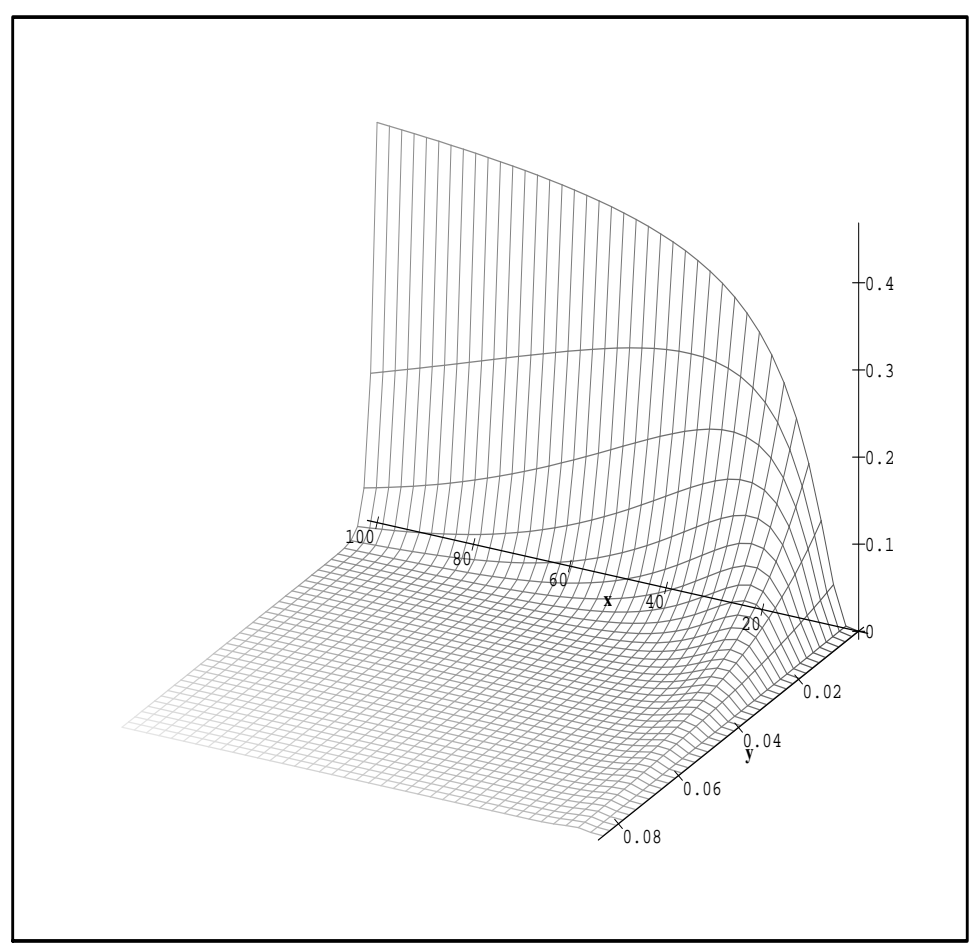

Figure 1: $|R(k)|^{2}$ plotted as a function of $\theta$. The notation $x=k$ and $y=\theta$ is used. For technical reasons the plot is made only from $\theta=10^{-5}$ instead of 0 . 